\title{
HAMILTONIAN FORMALISM OF BIANCHI TYPE I MODEL FOR PERFECT FLUID
}

\author{
Alokananda kar \\ $2^{\text {nd }}$ year M.Sc University of Calcutta ; Department of Physics; West Bengal ; India \\ Email : alokanandakar@gmail.com \\ Shouvik Sadhukhan \\ $2^{\text {nd }}$ year M.Sc Indian Institute of Technology ; Kharagpur ; Department of Physics ; West Bengal ; India \\ Email : shouvikphysics1996@gmail.com
}

\begin{abstract}
We propose the Hamiltonian formalism of Bianchi type 1 cosmological model for perfect fluid. We have considered both the equation of state parameter $\omega$ and the cosmological constant $\Lambda$ as the function of volume $V(t)$ which is defined by the product of three scale factors of the Bianchi type 1 line element. We propose a Lagrangian for the anisotropic Bianchi type-1 model in view of a variable mass moving in a variable potential. We can decompose the anisotropic expansion in terms of expansion and shearing motion by Lagrangian mechanism. We have considered a canonical transformation from expanding scale factor to scalar field $\varnothing$ which helps us to give the proper classical definition of that scalar field in terms of scale factors of the mentioned model. This definition helps us to explain the cosmological inflation. We have used large anisotropy(as in the cases of Bianchi models) and proved that cosmic inflation is not possible in such large anisotropy. Therefore we can conclude that the extent of anisotropy is less in case of our universe. Otherwise the inflation theory which explained the limitations of Big Bang cannot be resolved.Part II is contained with some analysis of the lagrangian ; derived in Part I ; on the quintessence model.
\end{abstract}

Keywords : General theory of Relativity ; Bianchi Type I model ; Isotropic and Anisotropic cosmology ; Perfect fluid ; Fluid mechanics ; Quintessence model ; cosmological inflation ; Viscosity ; Gravitational physics

\section{Introduction}

The accepted model of present day universe is homogenous and isotropic on large scales and is defined by the FRW model. However in the present high precision cosmology era there are certain experimental evidences of the broken isotropy of cosmic microwave background(CMB) in locar frame.The most common anisotropic expanding universe is explained by the Bianchi models.Although the experimental datas collected from CMBR provides the proof of isotropy of the universe in large scale basis but the local anisotropy may give the possibilities of initial anisotropy in early universe. 
Bianchi models are the flat generalization of FRW cosmological model where the spatial expansion is considered anisotropic. The models have been developed from the Bianchi Classification of Lie Algebra and that is why it is named as Lie Classification. Here we will be discussing about the type 1 model. This model is designed as flat space with spatial anisotropy.

The paper is organized as follows. In section A we calculate the Ricci tensor as well as the equation of motion from the Bianchi type 1 line element. The overall expansion is in terms of volume which is defined by the product of three scale factors. We define a Lagrangian and a potential which are a function of volume. The Lagrangian corresponds to a system in terms of variable mass moving in a variable potential.

In section B we simplify the above problem by making a canonical transformation to obtain a system comprising of a mass moving in a scalar field $\phi$ and we define the Lagrangian in terms of a scalar field $\phi$ and obtain a harmonic oscillator solution at the end of the cosmological inflation.

Finally in section $\mathrm{C}$ we did the analysis of our result by comparing it with large anisotropic deviation and small anisotropic deviation and proved that the cosmological inflation is not possible in large anisotropic deviation and concluded that the scale factors used in Bianchi type I model should not have much difference. It's all about the area given in part-l.

In part-Il we have used that lagrangian into the Einstein Hilbert action to modify the Quintessence model. From this modification we have shown how the inflation condition breaks due to high degree of anisotropy. Finally we have assumed the low value of anisotropy to satisfy the inflation condition as well as slow roll mechanism. At the end we have derived the variable $\mathrm{G}$ concept for this anisotropic model.

\section{Part-I}

\section{Section - A}

Bianchi type 1 metric is

$d s^{2}=-d t^{2}+X^{2}(t) d x^{2}+Y^{2}(t) d y^{2}+Z^{2}(t) d z^{2}$

Einstein Field equation states

$\mathrm{R}_{\mathrm{ab}}-\frac{1}{2} \mathrm{R} \mathrm{g}_{\mathrm{ab}}+\wedge \mathrm{g}_{\mathrm{ab}}=\mathrm{GT}_{\mathrm{ab}}$

For a perfect fluid the energy momentum tensor is given by

$T_{a b}=(p+\rho) u_{a} u_{b}+p g_{a b}$

For Bianchi Type 1 the field equations are

$(\dot{X} \dot{Y} / X Y)+(\dot{Z} \dot{Y} / Z Y)+(\dot{X} \dot{Z} / X Z)=G \rho+\Lambda$

$(\ddot{Y} / Y)+(\ddot{Z} / Z)+(\dot{Y} \dot{Z} / Y Z)=-G p+\Lambda$ 
$(\ddot{X} / X)+(\ddot{Z} / Z)+(\dot{Z} \dot{X} / Z X)=-G p+\Lambda$

$(\ddot{X} / X)+(\ddot{Y} / Y)+(\dot{X} \dot{Y} / X Y)=-G p+\Lambda$

Adding equation (5),(6) and (7) we get

$2\left(\left(\frac{\ddot{Y}}{Y}\right)+\left(\frac{\ddot{X}}{X}\right)+\left(\frac{\ddot{Z}}{Z}\right)\right)+\left(\left(\frac{\dot{X} \dot{Y}}{X Y}\right)+\left(\frac{\dot{Z} \dot{Y}}{Z Y}\right)+\left(\frac{\dot{Z} \dot{X}}{Z X}\right)\right)=-3 G p+3 \wedge$

The equation of state of the matter(cosmic fluid except the cosmological constant) is commonly assumed to be

$$
\mathrm{p}=(\gamma-1) \rho
$$

We combine equation (4),(8),(9) to get

$$
\left(\left(\frac{\ddot{Y}}{Y}\right)+\left(\frac{\ddot{X}}{X}\right)+\left(\frac{\ddot{Z}}{\mathrm{Z}}\right)\right)+\left(\frac{3 \gamma-2}{2}\right)\left(\left(\frac{\dot{X} \dot{Y}}{\overline{X Y}}\right)+\left(\frac{\dot{Z} \dot{Y}}{Z Y}\right)+\left(\frac{\dot{Z} \dot{X}}{Z X}\right)\right)-\frac{3 \gamma \Lambda}{2}=0
$$

We consider equation (10) as the basic starting point, if the dynamical equation for the scale factor can be written as that form , the present framework is valid. Our aim is to find a Hamiltonian description of equation (10) as the classical equation of motion. Now the volume dependent Lagrangian is ;-

$L_{V}=\frac{1}{2} M(V) \dot{V}^{2}-\tilde{V}(V) \quad \tilde{V}(V)=$ Variable Potential

And we consider a function $V$ which is defined by the product of three scale factors $\mathrm{V}=\mathrm{X}(\mathrm{t}) \mathrm{Y}(\mathrm{t}) \mathrm{Z}(\mathrm{t})$

The Euler Lagrangian Equation is

$\frac{d}{d t}\left(\frac{\partial \mathrm{Lv}}{\partial \dot{V}}\right)-\frac{\partial \mathrm{Lv}}{\partial V}=0 ;$

Or; $\frac{\ddot{V}}{V}-\frac{1}{2} \frac{\partial \ln M}{\partial \ln V}\left(\frac{\dot{V}}{V}\right)^{2}+(1 / \mathrm{MV})\left(\frac{\partial \tilde{\mathrm{V}}}{\partial V}\right)=0$

Now, $\frac{\dot{V}}{V}=\frac{\dot{X}}{X}+\frac{\dot{Y}}{Y}+\frac{\dot{Z}}{Z}$

$\frac{\ddot{V}}{V}=\left(\left(\frac{\ddot{Y}}{Y}\right)+\left(\frac{\ddot{X}}{\mathrm{X}}\right)+\left(\frac{\ddot{Z}}{\mathrm{Z}}\right)\right)+2\left(\left(\frac{\dot{X} \dot{Y}}{\mathrm{XY}}\right)+\left(\frac{\dot{Z} \dot{Y}}{Z Y}\right)+\left(\frac{\dot{Z} \dot{X}}{\mathrm{ZX}}\right)\right)$

We can rewrite equation (12) after using these equations we get

$$
\begin{aligned}
& \left(\left(\frac{\ddot{Y}}{Y}\right)+\left(\frac{\ddot{X}}{\mathrm{X}}\right)+\left(\frac{\ddot{Z}}{\mathrm{Z}}\right)\right)+2\left(1-\frac{3}{4} \frac{\partial \ln M}{\partial \ln V}\right)\left(\left(\frac{\dot{X} \dot{Y}}{\mathrm{XY}}\right)+\left(\frac{\dot{Z} \dot{Y}}{Z Y}\right)+\left(\frac{\dot{Z} \dot{X}}{\mathrm{ZX}}\right)\right)+(1 / \mathrm{MV})\left(\frac{\partial \tilde{\mathrm{V}}}{\partial V}\right)-\frac{1}{2} \frac{\partial \ln M}{\partial \ln V}\left(\left(\frac{\dot{X}}{X}\right)^{2}+\left(\frac{\dot{Y}}{Y}\right)^{2}+\left(\frac{\dot{Z}}{Z}\right)^{2}-\right. \\
& \left.\left(\left(\frac{\dot{X} \dot{Y}}{\mathrm{XY}}\right)+\left(\frac{\dot{Z} \dot{Y}}{Z Y}\right)+\left(\frac{\dot{Z} \dot{X}}{\mathrm{ZX}}\right)\right)\right)=0---(13)
\end{aligned}
$$


Comparing equation (10) and (13) we get

$2\left(1-\frac{3}{4} \frac{\partial \ln M}{\partial \ln V}\right)=\left(\frac{3 \gamma-2}{2}\right)$

$\mathrm{M}=\exp \int \frac{2-\gamma}{V} \mathrm{dV}$

$(1 / \mathrm{MV})\left(\frac{\partial \tilde{\mathrm{v}}}{\partial V}\right)=-\frac{3 \gamma \Lambda}{2}$

$\tilde{\mathrm{v}}=-\frac{3}{2} \int M V \gamma \Lambda \mathrm{dV}$

Here we are getting an extra part in the equation 13. We have already said in equation 11 that our considered lagrangian is dependent upon the volume expansion only and the euler lagrangian equation giving us the extra part when we want to compare this with equation 10 , the extra part is as ;-

$\frac{1}{2} \frac{\partial \ln M}{\partial \ln V}\left(\left(\frac{\dot{X}}{X}\right)^{2}+\left(\frac{\dot{Y}}{Y}\right)^{2}+\left(\frac{\dot{Z}}{Z}\right)^{2}-\left(\left(\frac{\dot{X} Y}{X Y}\right)+\left(\frac{\dot{Z} \dot{Y}}{Z Y}\right)+\left(\frac{\dot{Z} \dot{X}}{\mathrm{ZX}}\right)\right)\right) \neq 0$; if $\mathrm{X} \neq \mathrm{Y} \neq \mathrm{Z}$

That means if the first part i.e ;

$\left(\left(\frac{\ddot{Y}}{\mathrm{Y}}\right)+\left(\frac{\ddot{X}}{\mathrm{X}}\right)+\left(\frac{\ddot{\mathrm{Z}}}{\mathrm{Z}}\right)\right)+2\left(1-\frac{3}{4} \frac{\partial \ln M}{\partial \ln V}\right)\left(\left(\frac{\dot{\mathrm{X}} \dot{\mathrm{Y}}}{\mathrm{XY}}\right)+\left(\frac{\dot{\mathrm{Z}} \dot{\mathrm{Y}}}{Z Y}\right)+\left(\frac{\dot{\mathrm{Z}} \dot{\mathrm{X}}}{\mathrm{ZX}}\right)\right)+(1 / \mathrm{MV})\left(\frac{\partial \tilde{\mathrm{v}}}{\partial V}\right)=0$;

Then the euler lagrangian equation of motion will not give zero that means ;

$$
\begin{aligned}
& \left(\left(\frac{\ddot{Y}}{Y}\right)+\left(\frac{\ddot{X}}{\mathrm{X}}\right)+\left(\frac{\ddot{Z}}{\mathrm{Z}}\right)\right)+2\left(1-\frac{3}{4} \frac{\partial \ln M}{\partial \ln V}\right)\left(\left(\frac{\dot{X} \dot{Y}}{\mathrm{XY}}\right)+\left(\frac{\dot{Z} \dot{Y}}{Z Y}\right)+\left(\frac{\dot{Z} \dot{X}}{\mathrm{ZX}}\right)\right)+(1 / \mathrm{MV})\left(\frac{\partial \tilde{\mathrm{V}}}{\partial V}\right)-\frac{1}{2} \frac{\partial \ln M}{\partial \ln V}\left(\left(\frac{\dot{X}}{X}\right)^{2}+\left(\frac{\dot{Y}}{Y}\right)^{2}+\left(\frac{\dot{Z}}{Z}\right)^{2}-\right. \\
& \left.\left(\left(\frac{\dot{X} \dot{Y}}{\mathrm{XY}}\right)+\left(\frac{\dot{\mathrm{Z}} \dot{Y}}{Z Y}\right)+\left(\frac{\dot{Z} \dot{X}}{\mathrm{ZX}}\right)\right)\right) \neq 0
\end{aligned}
$$

So we have some deficiency in the consideration of the lagrangian in equation 11 and we have to add some extra part that mutually make all the extra terms as zero.

So suppose the new modified lagrangian is

$L=L_{v}+L_{G}$

Where the extra term $L_{G}$ is the lagrangian to modify the system motion definition which is also a function of $V$ and $\dot{V}$. So from the euler lagrangian equation using the modified lagrangian, we get ;-

$\frac{d}{d t}\left(\frac{\partial L}{\partial \dot{V}}\right)-\frac{\partial L}{\partial V}=\frac{d}{d t}\left(\frac{\partial \mathrm{Lg}}{\partial \dot{V}}\right)-\frac{\partial \mathrm{Lg}}{\partial V}+\frac{d}{d t}\left(\frac{\partial \mathrm{Lv}}{\partial \dot{V}}\right)-\frac{\partial \mathrm{LV}}{\partial V}=\frac{d}{d t}\left(\frac{\partial \mathrm{LV}}{\partial \dot{V}}\right)-\frac{\partial \mathrm{LV}}{\partial V}+\mathrm{Q}$

Where $\mathrm{Q}=\frac{d}{d t}\left(\frac{\partial \mathrm{Lg}}{\partial \dot{V}}\right)-\frac{\partial \mathrm{Lg}}{\partial V}$

The Euler Lagrangian equation corresponding to equation (14) is 
$\frac{\ddot{V}}{V}-\frac{1}{2} \frac{\partial \ln M}{\partial \ln V}\left(\frac{\dot{V}}{V}\right)^{2}+(1 / \mathrm{MV})\left(\frac{\partial \tilde{v}}{\partial V}\right)+\left(\frac{Q}{M V}\right)=0$

Now, $\frac{\dot{V}}{V}=\frac{\dot{X}}{X}+\frac{\dot{Y}}{Y}+\frac{\dot{Z}}{Z}$

$\frac{\ddot{V}}{V}=\left(\left(\frac{\ddot{Y}}{\mathrm{Y}}\right)+\left(\frac{\ddot{\mathrm{X}}}{\mathrm{X}}\right)+\left(\frac{\ddot{\mathrm{Z}}}{\mathrm{Z}}\right)\right)+2\left(\left(\frac{\dot{\mathrm{X}} \dot{Y}}{\mathrm{XY}}\right)+\left(\frac{\dot{\mathrm{Z}} \dot{Y}}{Z Y}\right)+\left(\frac{\dot{\mathrm{Z}} \dot{\mathrm{X}}}{\mathrm{ZX}}\right)\right)$

We can rewrite equation (15) after using these equations we get

$$
\begin{aligned}
& \left(\left(\frac{\ddot{Y}}{\mathrm{Y}}\right)+\left(\frac{\ddot{X}}{\mathrm{X}}\right)+\left(\frac{\ddot{Z}}{\mathrm{Z}}\right)\right)+2\left(1-\frac{3}{4} \frac{\partial \ln M}{\partial \ln V}\right)\left(\left(\frac{\dot{\mathrm{X}} \dot{Y}}{\mathrm{XY}}\right)+\left(\frac{\dot{\mathrm{Z}} \dot{Y}}{Z Y}\right)+\left(\frac{\dot{\mathrm{Z}} \dot{\mathrm{X}}}{\mathrm{ZX}}\right)\right)+\frac{Q}{M V}+(1 / \mathrm{MV})\left(\frac{\partial \tilde{\mathrm{v}}}{\partial V}\right)- \\
& \frac{1}{2} \frac{\partial \ln M}{\partial \ln V}\left(\left(\frac{\dot{X}}{X}\right)^{2}+\left(\frac{\dot{Y}}{Y}\right)^{2}+\left(\frac{\dot{Z}}{Z}\right)^{2}-\left(\left(\frac{\dot{X} \dot{Y}}{\mathrm{XY}}\right)+\left(\frac{\dot{\mathrm{Z}} \dot{Y}}{Z Y}\right)+\left(\frac{\dot{\mathrm{Z}} \dot{\mathrm{X}}}{\mathrm{ZX}}\right)\right)\right)=0---(16)
\end{aligned}
$$

So we get ;-

$\frac{Q}{M V}-\frac{1}{2} \frac{\partial \ln M}{\partial \ln V}\left(\left(\frac{\dot{X}}{X}\right)^{2}+\left(\frac{\dot{Y}}{Y}\right)^{2}+\left(\frac{\dot{Z}}{Z}\right)^{2}-\left(\left(\frac{\dot{X} \dot{Y}}{X Y}\right)+\left(\frac{\dot{Z} \dot{Y}}{Z Y}\right)+\left(\frac{\dot{Z} \dot{X}}{Z X}\right)\right)\right)=0$

$\mathrm{Q}=\frac{M V}{2} \frac{\partial \ln M}{\partial \ln V} \sigma^{2}$

Where $\sigma$ is the shearing scalar given by

$\sigma^{2}=\left(\frac{\dot{X}}{X}\right)^{2}+\left(\frac{\dot{Y}}{Y}\right)^{2}+\left(\frac{\dot{Z}}{Z}\right)^{2}-\left(\left(\frac{\dot{X} \dot{Y}}{X Y}\right)+\left(\frac{\dot{Z} \dot{Y}}{Z Y}\right)+\left(\frac{\dot{Z} \dot{X}}{Z X}\right)\right)$

From equation (16) it is evident that we get a shearing in the cosmic fluid due to the anisotropy present in Bianchi type 1 model .

So finally we get a proper definition of the extra lagrangian term $L_{G}$ which is giving the shearing on the system motion. But the problem is that we have considered $\mathrm{L}_{\mathrm{g}}$ as a function of $\mathrm{V}$ and $\dot{V}$ so we have to show the the shearing scalar is also a function of same.

We know that $X \neq Y \neq Z$ again we know;

$\frac{\dot{V}}{V}=\frac{\dot{X}}{X}+\frac{\dot{Y}}{Y}+\frac{\dot{Z}}{Z}$

Now let $\Delta_{1} ; \Delta_{2}$ and $\Delta_{3}$ are three excess term for the scale factors that make them different from $\sqrt[3]{V}$. so we may write ;

$\frac{1}{3} \frac{\dot{V}}{V}=\frac{\dot{X}}{X}+\frac{\dot{\Delta}_{1}}{\Delta_{1}} \quad ; \quad \frac{1}{3} \frac{\dot{V}}{V}=\frac{\dot{Y}}{Y}+\frac{\dot{\Delta}_{2}}{\Delta_{2}} \quad ; \quad \frac{1}{3} \frac{\dot{V}}{V}=\frac{\dot{Z}}{Z}+\frac{\dot{\Delta}_{3}}{\Delta_{3}}$

Where $\frac{\dot{\Delta}_{1}}{\Delta_{1}}+\frac{\dot{\Delta}_{2}}{\Delta_{2}}+\frac{\dot{\Delta}_{3}}{\Delta_{3}}=0$

Now from equation 18 by solving them we get; 
$\mathrm{X}=\frac{V^{1 / 3}}{\Delta_{1}} \quad ; \quad \mathrm{Y}=\frac{V^{1 / 3}}{\Delta_{2}} \quad ; \quad \mathrm{Z}=\frac{V^{1 / 3}}{\Delta_{3}}$

Now from the equation 17a we can write as ;

$$
\begin{aligned}
& \sigma^{2}=\left(\frac{1}{3} \frac{\dot{V}}{V}-\frac{\dot{\Delta}_{1}}{\Delta_{1}}\right)^{2}+\left(\frac{1}{3} \frac{\dot{V}}{V}-\frac{\dot{\Delta}_{2}}{\Delta_{2}}\right)^{2}+\left(\frac{1}{3} \frac{\dot{V}}{V}-\frac{\dot{\Delta}_{3}}{\Delta_{3}}\right)^{2}-\left(\left(\frac{1}{3} \frac{\dot{V}}{V}-\frac{\dot{\Delta}_{1}}{\Delta_{1}}\right)\left(\frac{1}{3} \frac{\dot{V}}{V}-\frac{\dot{\Delta}_{2}}{\Delta_{2}}\right)+\left(\frac{1}{3} \frac{\dot{V}}{V}-\frac{\dot{\Delta}_{2}}{\Delta_{2}}\right)\left(\frac{1}{3} \frac{\dot{V}}{V}-\frac{\dot{\Delta}_{3}}{\Delta_{3}}\right)+\right. \\
& \left.\left(\frac{1}{3} \frac{\dot{V}}{V}-\frac{\dot{\Delta}_{3}}{\Delta_{3}}\right)\left(\frac{1}{3} \frac{\dot{V}}{V}-\frac{\dot{\Delta}_{1}}{\Delta_{1}}\right)\right)
\end{aligned}
$$

so it is proved that the shearing scalar is also a function of $\mathrm{V}$ and $\dot{V}$. So the $\mathrm{Q}$ is also a function of them.

So from equation $17 ; 17 a ; 14 ; 14 a$ we can conclude that the lagrangian mechanism decomposed the anisotropic expanding motion of the cosmological model into it's expansion and shearing motion.

So finally the lagrangian will be as follows;-

$\mathrm{L}=\frac{1}{2} \mathrm{M}(\mathrm{V}) \dot{\mathrm{V}}^{2}-\tilde{\mathrm{V}}(\mathrm{V})+L_{g}$

Where $M(V)$ and $\tilde{v}(V)$ are the variable mass and the variable potential respectively and they are variable of volume factor $\mathrm{V} . L_{g}$ is the lagrangian to represent the shearing and $\mathrm{Q}=\frac{d}{d t}\left(\frac{\partial \mathrm{Lg}}{\partial \dot{V}}\right)-\frac{\partial \mathrm{Lg}}{\partial V}$ and also $\mathrm{Q}=\frac{M V}{2} \frac{\partial \ln M}{\partial \ln V} \sigma^{2}$ and $\sigma^{2}=\left(\frac{\dot{X}}{X}\right)^{2}+\left(\frac{\dot{Y}}{Y}\right)^{2}+\left(\frac{\dot{Z}}{Z}\right)^{2}-\left(\left(\frac{\dot{X} \dot{Y}}{\mathrm{XY}}\right)+\left(\frac{\dot{Z} \dot{Y}}{Z Y}\right)+\left(\frac{\dot{Z} \dot{X}}{\mathrm{ZX}}\right)\right)$. So we can able to distribute the lagrangian for bianchi type I universe into volume expanding and shearing part using lagrangian mechanism.

\section{Section - B}

Now it is the time to get the canonical transformation to give a definition of the scalar field in classical point of view.

Let consider a generalized equation that can represent the anisotropic equation given in equation 15 ;-

$\ddot{q}=\mathrm{f}_{1}(\mathrm{q}) \dot{q}^{2}+\mathrm{f}_{2}(\mathrm{q})+\mathrm{f}_{3}(\mathrm{q}, \dot{q})$

$f_{1}(q), f_{2}(q), f_{3}$ are specified functions, $f_{3}$ is due to anisotropy. Here $q$ is equivalent to $V$.

Comparing equation (15) and (21) we get

$M=\exp \left[-2 \int f_{1}(q) d q\right]$

$\mathrm{Q}=\mathrm{Mf}_{3}$ 
$\tilde{v}=-\int M_{2}(q) d q$

Now we consider a new variable $\phi$ as $d \phi=\exp \left[-\int f_{1}(q) d q\right] d q$

This transformation can be used to write the Lagrangian in terms of new variable $\phi$

It is evident that $\dot{\varphi}^{2}=\mathrm{M} \dot{q}^{2}$

,$\frac{1}{2} \mathrm{M} \dot{q}^{2}=\frac{1}{2} \dot{\varphi}^{2}$

Now, $\frac{d \varphi}{d t}=\exp \left[-\int \mathrm{f}_{1}(\mathrm{q}) \mathrm{dq}\right] \frac{d q}{d t}=\dot{\varphi}$

$\frac{d \dot{\varphi}}{d t}=\exp \left[-\int f_{1}(q) d q\right]\left[\ddot{q}-f_{1}(q) \dot{q}^{2}\right]$

$\ddot{\varphi}=\left[f_{3}+f_{2}\right] \exp \left[-\int f_{1}(q) d q\right]$

(23) (using equation (21)

So we have defined the scalar field using the generalized scale factor which gives the definition of scalar field in terms of volume expansion.

Now the lagrangian in terms of scaler field will become ;-

$\mathrm{L}=\frac{1}{2} \dot{\varphi}^{2}-\tilde{\mathrm{V}}(\phi)+\mathrm{Lg}$

Here $L_{g}$ is the function of $\phi . V$ or $q$ is the function of $\phi$ and $L_{g}$ is a function of $V$ and $\dot{V}$ so we can say that $\mathrm{L}_{\mathrm{g}}$ is a function of $\varnothing$ and $\dot{\varnothing}$.

The Euler Lagrangian equation becomes $\ddot{\varphi}+\frac{\partial \tilde{\mathrm{v}}}{\partial \varphi}=-\frac{d}{d t}\left(\frac{\partial \mathrm{Lg}}{\partial \dot{\varphi}}\right)+\frac{\partial \mathrm{Lg}}{\partial \varphi}$

$\ddot{\varphi}=-\frac{d}{d t}\left(\frac{\partial \mathrm{Lg}}{\partial \dot{\varnothing}}\right)+\frac{\partial \mathrm{Lg}}{\partial \varnothing}-\frac{\partial \tilde{\mathrm{v}}}{\partial \varphi}=\left[\mathrm{f}_{3}+\mathrm{f}_{2}\right] \exp \left[-\int \mathrm{f}_{1}(\mathrm{q}) \mathrm{dq} \quad\right.$ (using equation (21)

So we can write from the previous equation that

$\tilde{v}=-\int f_{2}(q) \exp \left[-2 \int f_{1}(q) d q\right] d q$

And $-\frac{d}{d t}\left(\frac{\partial \mathrm{Lg}}{\partial \dot{\varnothing}}\right)+\frac{\partial \mathrm{Lg}}{\partial \varnothing}=\mathrm{f}_{3} \exp \left[-\int \mathrm{f}_{1}(\mathrm{q}) \mathrm{dq}=\mathrm{Q}_{1}\right.$

Now $Q d q=Q_{1} d \phi=f_{3} \exp \left[-2 \int f_{1}(q) d q\right] d q$

$\mathrm{Q}=\mathrm{Q}_{1} \frac{d \emptyset}{d q}=\left[-\frac{d}{d t}\left(\frac{\partial \mathrm{Lg}}{\partial \dot{\emptyset}}\right)+\frac{\partial \mathrm{Lg}}{\partial \emptyset}\right] \frac{d \emptyset}{d q}$

Hence the Lagrangian in terms of scalar field is $L=\frac{1}{2} \dot{\varphi}^{2}-\tilde{V}(\phi)+\mathrm{Lg}$

So we get the canonical lagrangian for anisotropic cosmology and it can be used in Einstein Hilbert action to modify the quintessence model.

In equation we have shown the transformation of $Q$ into the function of scalar field.From this equation we can get the $\mathrm{L}_{\mathrm{g}}$ in as a function of scalar field by inverse euler equation derivation.

Using the $\mathrm{V}$ instead of $q$ we get ; 
As we know $\phi=\int \sqrt{M} \mathrm{dV}$

$\phi=\int \exp \left(\int \frac{2-\gamma}{2 V} \mathrm{dV}\right) \mathrm{dV}$ -(24)(using equation 13a)

By comparing (21a) and (13a),(13b)

We get $-2 \mathrm{f}_{1}=\frac{2-\gamma}{V}$

$$
\begin{aligned}
& f_{1}=-\left(\frac{2-\gamma}{2 V}\right) \\
& f_{2}=\frac{3 \gamma V \Lambda}{2}
\end{aligned}
$$

if Both $\Lambda, \gamma$ are both constants then the equation (24) gives us

$\phi=\frac{2}{4-\gamma} V^{(4-\gamma) / 2}$ for $\gamma \neq 0 ;$ and $\quad \phi=1 / 2 V^{2} \quad$ for $\gamma=0$

so we see that for $\gamma=0$ or $w=-1 ; \phi$ does not give the exponential representation.

We can find the potential (taking $q$ instead of $\mathrm{V}$ )

$\tilde{v}(\phi)=-\int f_{2}(q) \exp \left[-2 \int f_{1}(q) d q\right] d q$

putting the values of $\mathrm{f}_{1}$ and $\mathrm{f}_{2}$ and integrating we get $\tilde{\mathrm{v}}=\frac{3 \gamma \Lambda}{2(\gamma-4)} V^{(4-\gamma)}$

Using the value of $\phi$ we can write $\tilde{v}(\phi)=\frac{3}{8} \gamma \Lambda \phi^{2}(\gamma-4)$ or we can write as

$\tilde{\mathrm{v}}(\phi)=\frac{3}{8} \gamma^{2} \Lambda \varphi^{2}-\frac{3}{2} \gamma \Lambda \varphi^{2}$ which is a distorted potential of harmonic oscillator.

Finally we can write the Lagrangian as $L=\frac{1}{2} \dot{\varphi}^{2}-\frac{3}{8} \gamma \Lambda \phi^{2}(\gamma-4)$

Therefore we get the Lagrangian of a distorted harmonic oscillator.

Equation 26 may also be written as $L=1 / 2(\nabla \varphi)^{2}-\mathrm{V}(\phi)+L_{g}$ where the potential has taken as $\mathrm{V}$ $(\phi)$ and the scaler field is a function of not only time but also the whole four coordinate system and thus the term $\dot{\varphi}^{2}$ has been substituted by generalized gradient $(\nabla \varphi)^{2}$.

\section{Section - C}

If we consider the case of $\gamma=0$, the equation of state parameter becomes $\omega=p / \rho=-1$, which corresponds to the time of cosmological inflation .But in case of anisotropic universe we do not get the exponential expansion of universe as $\phi=1 / 2 V^{2}$ (or $\phi=1 / 2 q^{2}$ ) for $\gamma=0$ during at $w=-1$. So for anisotropy we are not getting inflation that may break the solutions of big bang cosmology and we must have to follow the inflation. 
Therefore, We can assume that during the time of cosmological inflation the expansion of the universe was so rapid that it was almost isotropic(if the anisotropy in expansion is small). One such expanding isotropic model is FRW model. So for $\gamma=0$ we can assume the universe to satishfy FRW model(with zero curvature). For which the scaler field is given by

$\phi=\int \exp \left(\int\left(\frac{3 \gamma-2}{2 q}\right) d q\right) d q$

For $\gamma=0$, we get $\phi=\ln (q)$ which satisfies the exponential expansion of universe during cosmological expansion.

\section{Part-II}

We have discussed here the quintessence model i.e the model with canonical lagrangian and kinetic energies.In section A ; we have discussed the quintessence model shortly for FRW model in perfect fluid in reference to the publication by Edmund J Copeland; M.sami, and shinji Tsujikawa. In this section we have given the action with canonical lagrangian for isotropic and homogeneous universe. In section B we have used the canonical lagrangian for anisotropic and homogeneous universe and taken the lagrangian from PART - I . In section C finally we have used the divergenceless condition of energy-momentum tensor of perfect fluid with anisotropic cosmology and proved that the gravitational constant should vary inversely with time to stabilize the universe with high value of anisotropy in cosmological model.

\section{Section A}

Quintessence is described by an ordinary scalar field $\phi$ which is a function of time, but we will see with particular potentials that lead to late time inflation. The action for Quintessence is given by

$\mathrm{S}=\int d^{4} x \sqrt{-g}\left[1 / 2(\nabla \varphi)^{2}-\mathrm{V}(\phi)\right]$

where $(\nabla \varphi)^{2}=g^{\mu \nu} \partial_{\mu} \varphi \partial_{v} \phi$ and $\vee(\phi)$ is the potential of the field. In a flat FRW spacetime the variation of the action with respect to $\phi$ gives

$\ddot{\varphi}+3 \mathrm{H} \dot{\varphi}+\mathrm{dV} / \mathrm{d} \phi=0$

The energy momentum tensor of the field is derived by the action in terms of $g^{\mu \nu}$ :

$T_{\mu \nu}=-\frac{2}{\sqrt{-g}} \frac{\delta S}{\delta g^{\mu \nu}}$

We can write that $\delta \sqrt{-g}=-(1 / 2) \sqrt{-g} g_{\mu \nu} \delta g^{\mu \nu}$, then 
$T_{\mu \nu}=\partial_{\mu} \varphi \partial_{\nu} \phi-g_{\mu \nu}\left[1 / 2 g^{\alpha \beta} \partial_{\alpha} \varphi \partial_{\beta} \phi+\mathrm{V}(\phi)\right]$

In the flat Friedmann background we obtain the energy density and pressure density of the scalar field:

$\rho=-T_{0}^{0}=1 / 2 \dot{\varphi}^{2}+\mathrm{V}(\phi) \quad ; \quad p=T_{1}^{1}=1 / 2 \dot{\varphi}^{2}-\mathrm{V}(\phi)$

Then we get ;

$H^{2}=8 \pi \mathrm{G} / 3\left[1 / 2 \dot{\varphi}^{2}+\mathrm{V}(\phi)\right]$

$\frac{\ddot{a}}{a}=-8 \pi \mathrm{G} / 3\left[\dot{\varphi}^{2}-\mathrm{V}(\phi)\right.$

From v we get

$\mathrm{W}=\mathrm{p} / \rho=\frac{1 / 2 \dot{\varphi}^{2}-\mathrm{V}(\varphi)}{1 / 2 \dot{\varphi}^{2}+\mathrm{V}(\varphi)}$

From equation $\mathrm{Vb}$ if we get $\mathrm{V}(\phi)>>\dot{\varphi}^{2}$ then we can write

$\mathrm{W}=-1 \quad ; \quad \frac{w+1}{w-1}=0$

This is the condition for cosmological inflation.

\section{Section B}

In the previous section we have given the well known calculations for the cosmological inflation theory with Quintessence model. In the action we have used the canonical lagrangian for perfect fluid with isotropic and homogeneous cosmology. Now we will show how this result is changed for using the newly derived canonical lagrangian for anisotropic cosmology.

We know from the paper with title PART -I that the canonical lagrangian for the anisotropic cosmology is ;-

$\mathrm{L}=1 / 2(\nabla \varphi)^{2}-\mathrm{V}(\phi)+L_{g}$

So the action will become ;-

$\mathrm{S}=\int d^{4} x \sqrt{-g}\left[1 / 2(\nabla \varphi)^{2}-\mathrm{V}(\phi)+L_{g}\right]$

So using this action we get the energy momentum tensor as ;-

$T_{\mu \nu}=\partial_{\mu} \varphi \partial_{\nu} \phi-g_{\mu \nu}\left[1 / 2 g^{\alpha \beta} \partial_{\alpha} \varphi \partial_{\beta} \phi+\mathrm{V}(\phi)-L_{g}\right]+2 \frac{\partial L_{g}}{\partial g^{\mu \nu}}$

So ; using this equation (36) and from the concept of density and pressure in cosmology we get ;

$\rho=-T_{0}^{0}=1 / 2 \dot{\varphi}^{2}+\mathrm{V}(\phi)-L_{g}+2\left(\frac{\partial L_{g}}{\partial g^{\mu \nu}}\right)_{\mu=0 ; v=0} \quad ; \quad p=T_{1}^{1}=1 / 2 \dot{\varphi}^{2}-\mathrm{V}(\phi)+L_{g}+2\left(\frac{\partial L_{g}}{\partial g^{\mu \nu}}\right)_{\mu=1 ; v=1}$

so we get ; 


$$
\begin{aligned}
& H^{2}=8 \pi \mathrm{G} / 3\left[1 / 2 \dot{\varphi}^{2}+\mathrm{V}(\phi)-L_{g}+2\left(\frac{\partial L_{g}}{\partial g^{\mu \nu}}\right)_{\mu=0 ; v=0}\right] \\
& \frac{\ddot{a}}{a}=-8 \pi \mathrm{G} / 3\left[\dot{\varphi}^{2}-\mathrm{V}(\phi)+L_{g}+\left(\frac{\partial L_{g}}{\partial g^{\mu \nu}}\right)_{\mu=0 ; \nu=0}+3\left(\frac{\partial L_{g}}{\partial g^{\mu \nu}}\right)_{\mu=1 ; v=1}\right]
\end{aligned}
$$

From (v) we get

$$
\mathrm{W}=\mathrm{p} / \rho=\frac{1 / 2 \dot{\varphi}^{2}+\mathrm{V}(\varphi)-L_{g}+2\left(\frac{\partial L_{g}}{\partial g^{\mu v}}\right)_{\mu=0 ; v=0}}{1 / 2 \dot{\varphi}^{2}-\mathrm{V}(\varphi)+L_{g}+2\left(\frac{\partial L_{g}}{\partial g^{\mu v}}\right)_{\mu=1 ; v=1}}
$$

From equation( $38 \mathrm{~b}$ ) if we get $\mathrm{V}(\phi)>>\dot{\varphi}^{2}$ then we can write

$$
\mathrm{W}=\frac{\mathrm{V}(\varphi)-L_{g}+2\left(\frac{\partial L_{g}}{\partial g^{\mu \mathrm{V}}}\right)_{\mu=0 ; v=0}}{-\mathrm{V}(\varphi)+L_{g}+2\left(\frac{\partial L_{g}}{\partial g^{\mu \nu}}\right)_{\mu=1 ; v=1}} \quad ; \quad \frac{w+1}{w-1}=\frac{1}{\left(\mathrm{~V}(\varphi)-L_{g}\right)}\left[\frac{\left(\frac{\partial L_{g}}{\partial g^{\mu \nu}}\right)_{\mu=0 ; v=0}+\left(\frac{\partial L_{g}}{\partial g^{\mu \nu}}\right)_{\mu=1 ; v=1}}{\left(\frac{\partial g^{\mu \nu}}{\mu}\right)_{\mu=0 ; \nu=0}-\left(\frac{\partial L_{g}}{\partial g^{\mu \nu}}\right)_{\mu=1 ; v=1}}\right]---
$$

So from 38c we get ;

$\mathrm{W} \neq-1$ as well as $\frac{w+1}{w-1} \neq 0$

So we see that the inflation condition breaks if the values of $L_{g} ;\left(\frac{\partial L_{g}}{\partial g^{\mu \nu}}\right)_{\mu=0 ; \nu=0} ;\left(\frac{\partial L_{g}}{\partial g^{\mu \nu}}\right)_{\mu=1 ; \nu=1}$ are considerable in the system. So to get inflation condition those values should be too low to consider inflation. Thus we may say that during the inflation the anisotropy of the universe was too low and presently it is almost zero. But if we say the anisotropy of the universe is considerable in present universe ; then the scale during inflation was large. Again if we want to consider anisotropy then $\mathrm{G}$ must vary with time. We have given this proof in this following section.

\section{Section C}

From the Bianchi Type I model using the divergenceless condition of energy momentum tensor

$$
T_{\mu \nu}=\rho U_{\mu} U_{\nu}+\mathrm{p} h_{\mu \nu}
$$

We can write

$$
\dot{\rho}+(\rho+\mathrm{p})\left(\frac{\dot{X}}{X}+\frac{\dot{Y}}{Y}+\frac{\dot{Z}}{Z}\right)=0
$$

Or; $\dot{\rho}+3(\rho+p) H=0$

Now from 37 we get;

$$
\begin{aligned}
& \dot{\varphi} \ddot{\varphi}+\left(\frac{\partial V}{\partial \varphi}\right) \dot{\varphi}-\dot{\varphi} \frac{\partial L_{g}}{\partial \varphi}+2 \dot{\varphi} \frac{\partial}{\partial \varphi}\left(\frac{\partial L_{g}}{\partial g^{\mu \nu}}\right)_{\mu=0 ; \nu=0}+3 \mathrm{H}\left[\dot{\varphi}^{2}+2\left(\left(\frac{\partial L_{g}}{\partial g^{\mu \nu}}\right)_{\mu=0 ; v=0}+\left(\frac{\partial L_{g}}{\partial g^{\mu \nu}}\right)_{\mu=1 ; v=1}\right)\right]=0 \\
& \text { Or; } \ddot{\varphi}+3 H \dot{\varphi}+\frac{\partial V}{\partial \varphi}+2 \frac{\partial}{\partial \varphi}\left(\frac{\partial L_{g}}{\partial g^{\mu \nu}}\right)_{\mu=0 ; v=0}-\frac{\partial L_{g}}{\partial \varphi}+\frac{6 H}{\dot{\varphi}}\left(\left(\frac{\partial L_{g}}{\partial g^{\mu \nu}}\right)_{\mu=0 ; v=0}+\left(\frac{\partial L_{g}}{\partial g^{\mu \nu}}\right)_{\mu=1 ; \nu=1}\right)_{=0---(40)}
\end{aligned}
$$


Here $\dot{\varphi}$ act as friction in the second order differential equation.For inflation the potential should be flat and we can neglect the acceleration $\ddot{\varphi}$. For if the field $\phi$ starts off with a huge acceleration $\ddot{\varphi} \gg 1$, the friction term will take care of it.

So now if we apply the idea of slow roll mechanism we say that acceleration is huge and so ;

$3 \mathrm{H} \dot{\varphi}+\frac{\partial V}{\partial \varphi}+2 \frac{\partial}{\partial \varphi}\left(\frac{\partial L_{g}}{\partial g^{\mu \nu}}\right)_{\mu=0 ; \nu=0}-\frac{\partial L_{g}}{\partial \varphi}+\frac{6 H}{\dot{\varphi}}\left(\left(\frac{\partial L_{g}}{\partial g^{\mu \nu}}\right)_{\mu=0 ; \nu=0}+\left(\frac{\partial L_{g}}{\partial g^{\mu \nu}}\right)_{\mu=1 ; \nu=1}\right)=0$;

$\operatorname{Or} ; \dot{\varphi}^{2}+\frac{1}{3 H}\left[\frac{\partial V}{\partial \varphi}+2 \frac{\partial}{\partial \varphi}\left(\frac{\partial L_{g}}{\partial g^{\mu \nu}}\right)_{\mu=0 ; v=0}-\frac{\partial L_{g}}{\partial \varphi}\right] \dot{\varphi}+6\left(\left(\frac{\partial L_{g}}{\partial g^{\mu \nu}}\right)_{\mu=0 ; v=0}+\left(\frac{\partial L_{g}}{\partial g^{\mu \nu}}\right)_{\mu=1 ; v=1}\right)=0---(40 \mathrm{a})$

Now from the solution of this quadratic equation we can say that ;

If $\mathrm{A}=\frac{1}{3 H}\left[\frac{\partial V}{\partial \varphi}+2 \frac{\partial}{\partial \varphi}\left(\frac{\partial L_{g}}{\partial g^{\mu \nu}}\right)_{\mu=0 ; \nu=0}-\frac{\partial L_{g}}{\partial \varphi}\right]$;

$\mathrm{B}=6\left(\left(\frac{\partial L_{g}}{\partial g^{\mu \nu}}\right)_{\mu=0 ; v=0}+\left(\frac{\partial L_{g}}{\partial g^{\mu \nu}}\right)_{\mu=1 ; v=1}\right)$

Then we can write ;-

$\dot{\varphi}=\frac{-A \pm \sqrt{A^{2}-4 B}}{2} ;$

$\operatorname{Or} ; \dot{\varphi}=-\frac{1}{6 H}\left[\frac{\partial V}{\partial \varphi}+2 \frac{\partial}{\partial \varphi}\left(\frac{\partial L_{g}}{\partial g^{\mu \nu}}\right)_{\mu=0 ; v=0}-\frac{\partial L_{g}}{\partial \varphi}\right] \pm$

$\sqrt{\frac{1}{4}\left(\frac{1}{3 H}\left[\frac{\partial V}{\partial \varphi}+2 \frac{\partial}{\partial \varphi}\left(\frac{\partial L_{g}}{\partial g^{\mu \nu}}\right)_{\mu=0 ; \nu=0}-\frac{\partial L_{g}}{\partial \varphi}\right]\right)^{2}-6\left(\left(\frac{\partial L_{g}}{\partial g^{\mu \nu}}\right)_{\mu=0 ; \nu=0}+\left(\frac{\partial L_{g}}{\partial g^{\mu \nu}}\right)_{\mu=1 ; \nu=1}\right)}$

Now if $\partial_{\varphi} V=0 \quad$ and $\quad H^{2}=\mathrm{V} / 3$

Then we get ;

$\dot{\varphi}=-\frac{1}{6 H}\left[2 \frac{\partial}{\partial \varphi}\left(\frac{\partial L_{g}}{\partial g^{\mu \nu}}\right)_{\mu=0 ; \nu=0}-\frac{\partial L_{g}}{\partial \varphi}\right] \pm$

$\sqrt{\frac{1}{4}\left(\frac{1}{3 H}\left[2 \frac{\partial}{\partial \varphi}\left(\frac{\partial L_{g}}{\partial g^{\mu \nu}}\right)_{\mu=0 ; \nu=0}-\frac{\partial L_{g}}{\partial \varphi}\right]\right)^{2}-6\left(\left(\frac{\partial L_{g}}{\partial g^{\mu \nu}}\right)_{\mu=0 ; \nu=0}+\left(\frac{\partial L_{g}}{\partial g^{\mu \nu}}\right)_{\mu=1 ; \nu=1}\right)}$

Now again from equation 38 we can write it as; 
$\dot{\varphi}=-\frac{1}{6 \sqrt{8 \pi \mathrm{G} / 3\left[1 / 2 \dot{\varphi}^{2}+\mathrm{V}(\varphi)-L_{g}+2\left(\frac{\partial L_{g}}{\partial g^{\mu \nu}}\right)_{\mu=0 ; v=0}\right]}}\left[2 \frac{\partial}{\partial \varphi}\left(\frac{\partial L_{g}}{\partial g^{\mu \nu}}\right)_{\mu=0 ; v=0}-\frac{\partial L_{g}}{\partial \varphi}\right] \pm$

$\sqrt{\frac{1}{4}\left(\frac{1}{3 \sqrt{8 \pi \mathrm{G} / 3\left[1 / 2 \dot{\varphi}^{2}+\mathrm{V}(\varphi)-L_{g}+2\left(\frac{\partial L_{g}}{\partial g^{\mu \nu}}\right)_{\mu=0 ; \nu=0}\right]}}\left[2 \frac{\partial}{\partial \varphi}\left(\frac{\partial L_{g}}{\partial g^{\mu \nu}}\right)_{\mu=0 ; \nu=0}-\frac{\partial L_{g}}{\partial \varphi}\right)^{2}-6\left(\left(\frac{\partial L_{g}}{\partial g^{\mu \nu}}\right)_{\mu=0 ; \nu=0}+\left(\frac{\partial L_{g}}{\partial g^{\mu \nu}}\right)_{\mu=1 ; \nu=1}\right)\right.}$

Using the condition for slow roll mechanism we get ;

$\dot{\varphi}=-\frac{1}{6 \sqrt{8 \pi \mathrm{G} / 3\left[\mathrm{~V}(\varphi)-L_{g}+2\left(\frac{\partial L_{g}}{\partial g^{\mu \nu}}\right)_{\mu=0 ; v=0}\right]}}\left[2 \frac{\partial}{\partial \varphi}\left(\frac{\partial L_{g}}{\partial g^{\mu \nu}}\right)_{\mu=0 ; v=0}-\frac{\partial L_{g}}{\partial \varphi}\right] \pm$

$\sqrt{\frac{1}{4}\left(\frac{1}{3 \sqrt{8 \pi \mathrm{G} / 3\left[\mathrm{~V}(\varphi)-L_{g}+2\left(\frac{\partial L_{g}}{\partial g^{\mu \nu}}\right)_{\mu=0 ; \nu=0}\right]}}\left[2 \frac{\partial}{\partial \varphi}\left(\frac{\partial L_{g}}{\partial g^{\mu \nu}}\right)_{\mu=0 ; \nu=0}-\frac{\partial L_{g}}{\partial \varphi}\right)^{2}-6\left(\left(\frac{\partial L_{g}}{\partial g^{\mu \nu}}\right)_{\mu=0 ; \nu=0}+\left(\frac{\partial L_{g}}{\partial g^{\mu \nu}}\right)_{\mu=1 ; \nu=1}\right)\right.}$

So from this equation we see whether the degree of anisotropy is considerable and due to very low value of $\mathrm{G} ; \dot{\varphi}$ is becoming too high but at the starting of this calculation for slow roll mechanism it was already considered that this friction term is small and is not capable of changing $V(\varphi)$ significantly. So it seems this friction paremeter is considered small initially and showing very large value at the end. This is an ambiguity. It is better to consider the $G$ as a variable of time which has high value during inflation and decreases with increase of time. Thus this ambiguity will be resolved with small value of anisotropy in cosmological model.

\section{Conclusion}

- From the Bianchi 1 model we are getting two types of motion for the fluid and they are expansion and shearing. We are not getting rotation. Shearing comes as a separate part in the Lagrangian which acts as a perturbation.

- The cosmological fluid system under the Bianchi type 1 model can be considered as a body with variable mass $M$ (a function of volume) is moving in a variable potential $\vec{V}$ (a function of volume).

- The shearing do not modify the potential in which the body is moving, instead it gives a shear in the body geometry.

- The anisotropy of the universe should be low in value to get the cosmic inflation condition.So during inflation the coefficient $L_{g}$ tends to 0 . 
- If we consider the high degree of anisotropy, then the flatness problem, horizon problem and monopole problem can not be resolved with cosmological inflation concept.So we can say that universe can not be highly anisotropic.

- The gravitational constant should vary with time in inverse order in viscous universe i.e the value of this constant should be high during inflation and it should reduce as time increases to reach present time.

- If we consider that the gravitational constant does not change with time inversely, the slow roll model will fail to explain the cosmological inflation and that is impossible. So gravitational constant term $G$ should vary with time in case the universe is slightly anisotropic.

In this paper we have applied the perfect fluid condition. Our next aim is to do the same on bulk viscous fluid cosmology and anisotropic fluid cosmology. Also we will show how the quintessence model modifies using this lagrangian in Einstein-Hilbert action.

\section{Reference}

1. Hamiltonian formalism in friedmann cosmology and it's quantisation by JieRen ; Xin-He Meng ; and Liu Zhao [ 10.1103/physRev D.76.04351] e-print [ arXiv:0704.0672 [hep-th] ]

2. Bianchi type I cosmological models with variable $G$ and $\wedge$ by AroonkumarBeesham. [Beesham , A.GenRelatGravit (1994)26:159]

3. Bianchi type VI bulk viscous Fluid models with variable gravitational and cosmological constants by Manoj K Verma, Shri Ram.[ 10.4236/am.2011.23041]

4. The Energy-Momentum Tensor for a Dissipative Fluid in General RelativityOscar M. Pimentel - F. D. Lora-Clavijo Guillermo A. Gonz'alez[arxiv:1606.01318v2 14 $4^{\text {th }}$ September ]

5. Copeland, Edmund \& Sami, M. \& Tsujikawa, Shinji. (2006). Dynamics of dark energy. Int. J. Mod. Phys. D. 15.

6. Russell, Esra \& Kılınç,Can \& Pashaev, Oktay. (2013). Bianchi I Model: An Alternative Way To Model The Presentday Universe. Monthly Notices of the Royal Astronomical Society. 442. 10.1093/mnras/stu932

7. Dynamics of dark energy ;Edmund J. Copeland,M. Sami, and Shinji Tsujikawa

8. Inflation; Marieke Postma1; 1 NIKHEF, Science Park 1051098 XG Amsterdam, The Netherlands.

9. PHYSICAL REVIEW D 84, 024020 (2011) by Tiberiu Harko ; Francisco S.N. Lobo ; Shin'ichi Nojiri and Sergei D. Odintsov.

10. Slow roll inflation; Pascal Vaudrevange pascal@vaudrevange.com

11. A Scalar Field Theory for Dark Matter-Dark Energy Interaction ; Pedro Miguel Grego' rio Carrilho

12. THE QUINTESSENTIAL DARK ENERGY THEORY: QUINTESSENCE. ; A PREPRINT ;JackHughes Mathematical Physics University of Nottingham pmxjh7@nottingham.ac.uk

13. A quintessential introduction to dark energy By Paul J. Steinhardt Department of Physics, Princeton University, Princeton, NJ 08540, USA

14. The Energy-Momentum Tensor for a Dissipative Fluid in General Relativity ; Oscar M. Pimentel · F. D. Lora-Clavijo · Guillermo A. Gonz'alez

15. Sadhukhan. S, Quintessence Model Calculations for Bulk Viscous Fluid and Low Value Predictions of the Coefficient of Bulk Viscosity, International Journal of Science and Research (IJSR) 9(3):1419-1420, DOI: $10.21275 /$ SR20327132301

16. Kar. A, Sadhukhan. S, HAMILTONIAN FORMALISM FOR BIANCHI TYPE I MODEL FOR PERFECT FLUID AS WELL AS FOR THE FLUID WITH BULK AND SHEARING VISCOSITY, Basic and Applied Sciences into Next Frontiers, ISBN: 978-81-948993-0-3

17. SADHUKHAN, S.; KAR, A. Quintessence Model Calculations for Bulk viscous Fluid and Low Value Predictions of The coefficient of Bulk Viscosity in General as Well as Modified Gravity With the Form $F(R, T)=R+F(T)$ and $F(T)=\lambda T$.. Preprints 2020, 2020120775 (doi: 10.20944/preprints202012.0775.v1).

18. SADHUKHAN, S.; KAR, A. QUINTESSENCE MODEL WITH BULK VISCOSITY AND SOME PREDICTIONS ON THE COEFFICIENT OF BULK VISCOSITY AND GRAVITATIONAL CONSTANT.Recent Advancement of Mathematics in Science and Technology ; DOI: $\underline{10.13140 / R G .2 .2 .19900 .67202}$ 